\title{
Aux limites du prévisible
}

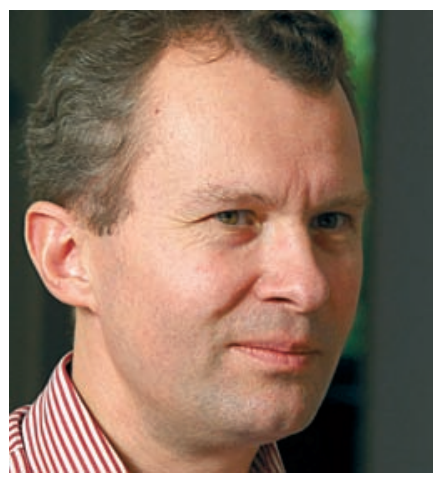

Christoph Rehmann-Sutter
Dans quelle mesure la médecine moderne contribue-t-elle à rendre notre vie plus prévisible, même si nous savons qu'en réalité rien ne peut véritablement être certain? Et comment, par le fait de lutter contre l'imprévisible, ouvret-elle la voie au hasard? Outre le fait qu'elle aide à guérir ou à apaiser les maux, la médecine estelle peut-être, rien que par effet secondaire, un moyen biotechnologique de limiter l'imprévisible, de modifier le destin de manière technologique et de réduire les éventualités?

Ce sont là des questions touchant à la philosophie de la médecine. Parfois également l'aspect religieux. Les religions ont toujours étudié avec grand intérêt le destin, la manière dont les êtres s'en accommodent, l'apprivoisent et saisissent les opportunités.

La médecine n'est pas une discipline de probabilités comme les prévisions météorologiques par exemple. Son éthique principale s'oriente sur l'assistance et non sur la prévision. Lorsque la médecine ne peut plus qu'annoncer sans intervenir, elle a atteint ses limites. Toutefois, dans certains domaines, le pronostic est essentiel. Par exemple lorsqu'il s'agit de tests génétiques prédictifs. Le diabète de type 2 , le cancer de la prostate seront bientôt sur la liste. Aujourd'hui déjà, elle contient beaucoup de maladies présentant un aspect génétique comme le cancer du sein, des ovaires ou de l'intestin ou des affections plus rares telles que la maladie de Marfan ou le syndrome de von Hippel-Lindau, etc. De par leur nature même, tous les diagnostiques prénataux sont également une entreprise pronostique. La question est: mon enfant sera-t-il en bonne santé? Mais la médecine établit aussi des pronostics dans d'autres domaines que la génétique. De nombreux risques sont connus à l'avance et l'évolution probable de notre fragile équilibre entre la santé et la maladie peut d'une certaine manière être calculé, du moins statistiquement, c'est-à-dire pour un groupe de personnes. Mais la question d'une personne reste toujours la même: que va-t-il m'arriver à moi? Que va-t-il lui arriver à lui ou à elle? En généralement, le calcul statistique n'apporte pas de réponse. Même dans les activités principales de la médecine, l'imprévisible diminue: on ne meurt plus aujourd'hui de nombreuses maladies qui auraient dans le temps été fatales. Il semble envisageable de planifier sa vie. La médecine aide les gens à avoir (ou à croire avoir) une «espérance de vie» biographique plus ou moins réaliste et souvent, à l'atteindre effectivement. Les décisions les plus importantes de notre existence dépendent toujours plus fréquemment de ces pronostics. Par exemple, il sera possible d'avoir des enfants plus tard. Lorsque la carrière aura évolué de manière satisfaisante, lorsque le partenaire idéal aura été trouvé! Mais il $\mathrm{y}$ a toujours des accidents et des drames parfois d'envergure (guerres, catastrophes naturelles, famines, changements écologiques avec des conséquences imprévisibles) et le destin nous frappe également souvent à l'improviste.

Ce serait d'ailleurs trop simple d'évaluer la possibilité de prévoir comme positive et l'impossibilité de prévoir comme négative. C'est en effet souvent l'imprévu qui génère les plus grands bonheurs. Où en serions-nous dans notre vie si nous pouvions tout organiser de manière impeccable? Que deviendraient toutes ces rencontres inopinées qui sont à la base de l'amour et de la famille? Aurions-nous encore ces moments inespérés de bonheur inédit?

La possibilité ou l'impossibilité de prévoir constitue un thème fascinant aux multiples aspects. Il s'intègre à la «culture» médicale. Pour le traiter, il faut une approche culturelle, artistique, littéraire, philosophie, religieuse et éthique de la médecine. Pour ce faire, l'Université de Zurich organise, avec le soutien de l'ASSM et de l'ASSH, un symposium interdisciplinaire international intitulé «Les limites du prévisible» qui se tiendra du 13 au 15 décembre 2007 [1].

Le prospectus du congrès est illustré par «L'édification de la Tour de Babel I» de Friedrich Dürrenmatt (1952): des êtres qui construisent des tours fragiles à des hauteurs vertigineuses, plus hautes que les montagnes. Ce sont des cathédrales de tours et les toits les plus hauts sont attachés avec des cordes dans une tentative désespérée d'éviter la chute. Qu'espèrent donc ces bâtisseurs? Le triomphe du calcul? Ou craignentils la chute qui suit inévitablement les hauteurs orgueilleuses? Où est la médecine dans ce tableau? Est-ce également une tour de ce type? Où se tient-elle au bas du bâtiment, avec des brancards et des services d'urgence pour le moment où les cordes lâcheront?

Maintenant que j'y ai pensé, ce thème va me tourner longtemps dans la tête.

Christoph Rehmann-Sutter* \footnotetext{
$\mathrm{du}$ BMS pour les questions d'éthique.

Dr ès lettres, biologiste diplômé, directeur du Service d'éthique dans les sciences biologiques de l versité de Bâle, président de la Commission nationale d'éthique
} 